\title{
The role of integrated mental and community physical healthcare trusts in responding to the COVID-19 pandemic in the UK
}

\author{
Derek Tracy, ${ }^{1}$ (1) Anna Forrest, ${ }^{2}$ Benjamin R. Underwood ${ }^{2}$ (1)
}

BJPsych Bulletin (2021) 45, 129-131, doi:10.1192/bjb.2020.61

\author{
'Oxleas NHS Foundation Trust, London, \\ UK; ${ }^{2}$ Fulbourn Hospital, Cambridgeshire \\ and Peterborough NHS Foundation \\ Trust, Cambridge, UK \\ Correspondence to Benjamin \\ R. Underwood (ben.underwood@cpft. \\ nhs.uk) \\ First received 28 Apr 2020, final revision \\ 11 May 2020, accepted 28 May 2020 \\ (C) The Authors 2020. This is an Open \\ Access article, distributed under the \\ terms of the Creative Commons \\ Attribution-NonCommercial-ShareAlike \\ licence (http://creativecommons.org/ \\ licenses/by-nc-sa/4.0/), which permits \\ non-commercial re-use, distribution, \\ and reproduction in any medium, \\ provided the same Creative Commons \\ licence is included and the original work \\ is properly cited. The written permission \\ of Cambridge University Press must be \\ obtained for commercial re-use.
}

Summary For several decades, mental health services within the UK's National Health Service were provided by specialist mental health trusts. More recently many of these trusts have integrated community physical health services into their operations. We describe here how two integrated mental health trusts in England were able to make an enhanced response to the COVID-19 pandemic.

Keywords Community mental health teams; economics; out-patient treatment; clinical governance; cost-effectiveness.
The pandemic of COVID-19 has been one of the biggest challenges to global healthcare in a century. This has been felt in the UK, where it has provided the greatest test in the history of its National Health Service (NHS). For nearly 20 years mental health services within the NHS have been provided by specialist mental health trusts. In more recent times these organisations have moved beyond mental health to increasingly include community physical health services. ${ }^{1}$ Much has been written on the role and response of NHS providers, and potential consequences for the service, in relation to mental health and COVID-19. ${ }^{2}$ However, COVID-19 is primarily a respiratory disease which carries the greatest risk for elderly people and this has created primarily a physical healthcare need in the early phases. ${ }^{3}$ In this editorial we describe the vital role of two integrated mental health trusts in England responding to the COVID-19 pandemic and discuss how the integration between mental and physical healthcare has enhanced this response.

\section{Background to the trusts}

Cambridgeshire and Peterborough NHS Foundation Trust (CPFT) serves a mixed, though predominantly rural, population of around 1000000 , including more than 165 000 people over the age of 65 . Originally formed as a mental health trust, for the past 5 years it has also been responsible for provision of community physical care for adults and older people in Cambridgeshire. This includes 102 rehabilitation in-patient beds, 3 minor injury units, district nursing, occupational therapy, speech and language therapy, physiotherapy, podiatry, and dietetics, in addition to specialist teams such as respiratory, cardiac, chronic fatigue, neurological rehabilitation, early supported discharge for stroke, epilepsy, Parkinson disease, multiple sclerosis, tissue viability and diabetes. These new services were merged with existing in-patient and community provision for older people's mental health to create an adult and older people's directorate, which includes urgent response to physical and psychiatric presentations in the community.

Oxleas NHS Foundation Trust (OFT) covers the southeast London boroughs of Bromley, Bexley and Greenwich, serving both inner- and outer-London urban populations. The Bexley directorate has an integrated structure known as 'Bexleycare' - incorporating NHS services for both mental health and community physical health with adult social care provided by the local authority. This is run by an integrated management team from health and 
social care, covering a large range of services for those over the age of 18. It has an in-patient site, Queen Mary's Hospital, that contains beds for both mental healthcare (working age adult, older persons' and dementia) and stepdown intermediate physical care. Community services for the boroughs' approximately 250000 residents include mental health services (for adults over >18 years of age), physical health services similar to those described for CPFT and adult social care.

\section{Changes in response to COVID-19}

In line with other NHS providers, CPFT entered a critical incident in March 2020 and adopted a command and control structure. This brought immediate changes to our physical and mental health services, including the formation of a county-wide COVID-19 testing service. From the perspective of physical health more generally we focused on the priority of preserving life. We felt we could best contribute to this by supporting discharge from acute trusts to allow patient flow and by treating people both with and without COVID-19 in the community to prevent deterioration and possible need for admission.

We created a single point of access and a telephone line that took 20000 calls in its first month of operation. We expanded our discharge to assess (DTA) team, which increased the number of patients it was seeing from 10-20 per day to up to 70 and dealt with 810 patients in its first month. Partly as a result of this, our local acute trusts were able to maintain bed availability. For our community teams we followed national guidance in terms of which services continued and which were suspended. A triage function with senior staff was created which acted on the principle of accepting referrals if the patient needed an intervention that would prevent deterioration likely to result in acute admission or was a threat to life. This principle-based approach was preferred to a list of which patients or conditions would be accepted. It required widespread redeployment of staff to enhance the most critical areas of service. For example, in older peoples' mental health, routine memory assessments were stopped and staff redeployed to in-patient care or crisis teams. We engaged with colleagues across the system to draw up new clinical guidelines. Perhaps the most notable example was with regard to end-of-life care for COVID-19 patients, where our community teams faced significant mortality, particularly among care home residents.

Bexleycare, as part of OFT, had both a trust-level and London-wide COVID-19 command response. Within Bexley, the joint governance under the NHS organisation and the local authority facilitated early work and communication with public health services (which fall under the local authority). Nursing services in the trust took the lead for infection control and testing, with district nursing services delivering this locally. In-patient mental health services were notably reduced, with a $50 \%$ reduction in bed base effected by intentional ward closure because of the risks presented to patients and staff. Intermediate physical health and dementia care wards were notable risk sites and, indeed, sadly there were early infections and deaths of patients on these wards.
Daily command calls linked the directorate with the trust and local authority, and through this, regional London command.

\section{Benefits of integration of physical and mental health services}

Across both trusts we found immediate and direct benefits of integration. Our mental health services have benefited from support from the physical health teams, for example having respiratory specialist nurses and consultant geriatricians to support our older people's mental health wards. Similarly, our physical health teams have benefited from an extensive psychological support package for staff to deal with what have been extreme circumstances at work. This has included a stepped approach to the support given (from advice on self-management, psychological first aid and signposting to one-to-one support and referral to specialist mental health support, if appropriate, for staff requiring additional help), access to a staff support telephone line delivered by psychology staff and the Staff Well-being Service, guidance for managers on looking after their staff's psychological well-being, and 'team time' sessions to help clinical teams to reflect on the personal impact of the work they are doing. These initiatives have helped promote compassionate leadership within our services and have been well received by staff. More generally, having a larger organisation has meant that there is a bigger pool of staff to draw from to bolster critical services, a particular advantage when facing potentially high levels of staff sickness. Some potential drawbacks, such as loss of specialism or identity, have been ameliorated by people coming together in a time of crisis.

\section{Conclusions}

Combined mental and physical healthcare trusts can change quickly and at scale in response to health emergencies. We have previously described the benefits of integrated trusts in times of normal business. ${ }^{1}$ Although integration does not hold all the answers or fully mitigate the current situation with COVID-19, we have seen that there are benefits in times of crisis and that these can have a positive effect on elements of the wider healthcare system.

\begin{abstract}
About the authors
Derek Tracy is a consultant psychiatrist and Clinical Director of Oxleas NHS Foundation Trust, London, UK; Anna Forrest is a consultant clinical psychologist at Fulbourn Hospital, Cambridgeshire and Peterborough NHS Foundation Trust, Cambridge, UK; Benjamin R. Underwood is a consultant psychiatrist and Deputy Medical Director at Fulbourn Hospital, Cambridgeshire and Peterborough NHS Foundation Trust, Cambridge, UK.
\end{abstract}

\section{Author contributions}

D.T. and B.R.U. created the concept for the article. D.T., A.F. and B.R.U. wrote and reviewed the text. 


\section{Declaration of interest}

None.

ICMJE forms are in the supplementary material, available online at https:// doi.org/10.1192/bjb.2020.61.

\section{References}

1 Tracy DK, Hanson K, Brown T, James AJB, Paulsen H, Mulliez Z, et al. Integrated care in mental health: next steps after the NHS Long Term Plan. Br J Psychiatry 2019; 214: 315-7.
2 Holmes EA, O'Connor RC, Perry VH, Tracey I, Wessely S, Arseneault L, et al. Multidisciplinary research priorities for the COVID-19 pandemic: a call for action for mental health science. Lancet Psychiatry 2020; 7: 547-60.

3 Goyal P, Choi JJ, Pinheiro LC, Schenck EJ, Chen R, Jabri A, et al. Clinical characteristics of Covid-19 in New York City. N Engl J Med [Epub ahead of print] 17 Apr, 2020. Available from: https://doi.org/10.1056/ NEJMc2010419.

\title{
EDITORIAL
}

\section{The effects of COVID-19 on self-harm in UK prisons}

\author{
Thomas Hewson, ${ }^{1}$ (1) Russell Green, ${ }^{2}$ Andrew Shepherd, ${ }^{3}$ Jake Hard, ${ }^{4}$ Jennifer Shaw ${ }^{3}$
}

BJPsych Bulletin (2021) 45, 131-133, doi:10.1192/bjb.2020.83

${ }^{1}$ Manchester University NHS Foundation Trust, UK; ${ }^{2}$ CareUK Healthcare, UK: ${ }^{3}$ University of Manchester, UK; ${ }^{4}$ Royal College of General Practitioners Secure Environments Group, UK

Correspondence to Dr Thomas Hewson (tomhewson@doctors.org.uk)

First received 8 Jun 2020, final revision 6 Jul 2020, accepted 8 Jul 2020

(c) The Author(s), 2020. Published by Cambridge University Press on behalf of BJPsych Bulletin. This is an Open Access article, distributed under the terms of the Creative Commons Attribution licence (http://creativecommons.org/ licenses/by/4.0/), which permits unrestricted re-use, distribution, and reproduction in any medium, provided the original work is properly cited.
Self-harm is a major international public health concern and is especially prevalent among prisoners. In this editorial, we explore recent trends in prisoner self-harm during the coronavirus lockdown, and consider strategies for improving the prevention and management of self-harm in prisons as we emerge from the pandemic.

Keywords Coronavirus; COVID-19; self-harm; prison; forensic mental health services.
The frequency and severity of self-harm have been increasing within UK prisons over recent years, with rates far exceeding those observed in the general population. ${ }^{1}$ We postulated that the coronavirus pandemic could adversely affect the mental health of prisoners and further increase rates of self-harm, given the rapid changes and reductions to prison regimes and the negative psychological effects of quarantine. ${ }^{2}$ However, internal reports from Safer Custody Units in 31 prisons where healthcare is provided by CareUK (R. Green, personal communication, 2020) have revealed fewer implementations of ACCT (assessment, care in custody and teamwork) processes since lockdown; these processes initiate care plans for prisoners at risk of self-harm or suicide. Across the 31 prisons, there were 1079 ACCTs implemented in February 2020, compared with 828 in April 2020, a relative reduction of just under $25 \%$. Furthermore, a closer analysis of eight of these prisons revealed overall reductions in recorded incidents of self-harm, decreasing by a third from 324 in
February 2020 to 214 in April 2020. There are many possible reasons for these apparent reductions, with important lessons to be learned.

The accurate recording and maintenance of ACCT processes is a legal requirement stipulated in Prison Service Instructions, ${ }^{3}$ supporting the reliability of the above figures. However, minor acts of self-harm may be more likely to be missed or unrecorded during the pandemic owing to potentially reduced face-to-face contact with prisoners and staffing issues. There have been national reports of fewer Accident \& Emergency attendances for various health problems, ${ }^{4}$ raising the possibility that prisoners have similarly been less likely to seek healthcare interventions for self-harm throughout the lockdown. A reduction in ACCTs is also not synonymous with reductions in self-harming behaviour, as multiple factors, including staff discretion, affect ACCT implementation; furthermore, Humber et al found that ACCTs were more likely to be opened following 